\title{
Objetivos de desarrollo sostenible y el control de enfermedades
}

\section{Sustainable development goals and disease control}

\author{
César Cabezas-Sánchez ${ }^{1, a}$ \\ ${ }^{1}$ Facultad de Medicina, Universidad Nacional Mayor de San Marcos. Lima, Perú. \\ ${ }^{a}$ Director, Anales de la Facultad de Medicina
}

\section{Correspondencia: \\ César Cabezas Sánchez \\ ccabezass@unmsm.edu.pe}

Recibido: 12 de junio 2018

Aprobado: 14 de junio 2018

Conflictos de interés: Ninguno.

Citar como: Cabezas-Sánchez C. Objetivos de desarrollo sostenible y el control de enfermedades [editorial]. An Fac med. 2018;79(2):113-4. DOI: http://dx.doi.org/10.15381/anales. v79i2.14581
El año 2000, 189 naciones del mundo formularon el plan denominado "Objetivos de Desarrollo del Milenio (ODM)" y se unieron para enfrentar el futuro que tenía que ver con hambrunas, sequías, guerras, plagas, pobreza y problemas crónicos en el mundo. Se sabía que teníamos suficiente comida para alimentar al mundo, pero que no estaba siendo compartida; que se tenían medicamentos para el VIH y para otras enfermedades, pero que eran muy costosos; que los terremotos y las inundaciones eran inevitables, pero no así la elevada cantidad de muertos. Se hicieron planes ambiciosos, como para erradicarlos en poco años. Surge entones en el año 2015 el acuerdo de las naciones por los Objetivos de Desarrollo Sostenible (ODS) ${ }^{1}$.

Estos ODS fueron 17 y la síntesis de 16 de ellos es que propusieron para el mundo: poner fin a la pobreza; al hambre; garantizar una educación inclusiva, equitativa y de calidad; lograr la igualdad entre los géneros; garantizar la disponibilidad de agua y saneamiento; garantizar el acceso a una energía segura, sostenible y moderna; promover el crecimiento económico sostenido, el empleo pleno y productivo; construir infraestructuras resilientes; promover la industrialización inclusiva; lograr que las ciudades y los asentamientos humanos sean inclusivos, seguros, resilientes y sostenibles; garantizar modalidades de consumo y producción sostenibles; adoptar medidas urgentes para combatir el cambio climático y sus efectos; conservar y utilizar en forma sostenible los océanos y los recursos marinos; proteger, restablecer y promover el uso sostenible de los ecosistemas terrestres, gestionar los bosques de forma sostenible, luchar contra la desertificación y poner freno a la pérdida de la diversidad biológica; y finalmente fortalecer los medios de ejecución y revitalizar la alianza mundial para el desarrollo sostenible?

El tercer objetivo de estos ODS fue el de garantizar una vida sana y promover el bienestar para todos en todas las edades. En este contexto, se consideró que para el año 2030 se debería reducir la tasa mundial de mortalidad materna a menos de 70 por cada 100,000 nacidos vivos; poner fin a las muertes evitables de recién nacidos y de niños menores de 5 años, logrando que todos los países intenten reducir la mortalidad neonatal al menos hasta 12 por cada 1,000 nacidos vivos, y la mortalidad de niños menores de 5 años al menos hasta 25 por cada 1,000 nacidos vivos; poner fin a las epidemias del SIDA, la tuberculosis, la malaria y las enfermedades tropicales desatendidas y combatir la hepatitis, las enfermedades transmitidas por el agua y otras enfermedades transmisibles; reducir en un tercio la mortalidad prematura por enfermedades no transmisibles mediante la prevención y el tratamiento; y promover la salud mental y el bienestar, fortaleciendo la prevención y el tratamiento del abuso de sustancias adictivas, incluido el uso indebido de estupefacientes y el consumo nocivo de alcohol; reducir a la mitad el número de muertes y lesiones causadas por accidentes de tráfico en el mundo; garantizar el acceso universal a los servicios de salud sexual y reproductiva, incluidos los de planificación de la familia, información y educación, y la integración de la salud reproductiva en las estrategias y los programas nacionales; lograr la cobertura sanitaria universal, en particular la protección contra los riesgos financieros, el acceso a servicios de salud esenciales de calidad y el acceso a medicamentos y vacunas seguros, eficaces, asequibles y de calidad para todos; reducir sustancialmente el número de muertes y enfermedades producidas por productos químicos peligrosos y la contaminación del aire, el agua y el suelo, fortalecer la aplicación del Convenio Marco de la Organización Mundial de la Salud para el Control del Tabaco en todos los países; según proceda, apoyar las actividades de investigación y desarrollo de vacunas y medicamentos para las enfermedades transmisibles y no transmisibles que afectan primordialmente a los países en desarrollo y facilitar el acceso a medicamentos y vacunas esenciales asequibles de conformidad con la Declaración de Doha relativa al Acuerdo sobre los Aspectos de los Derechos de Propiedad Intelectual relacionados al comercio y la salud pública, en la que se afirma el derecho 
de los países en desarrollo a utilizar al máximo las disposiciones del acuerdo en lo relativo a la flexibilidad para proteger la salud pública y, en particular, proporcionar acceso a los medicamentos para todos; aumentar sustancialmente la financiación de la salud y la contratación, el desarrollo, la capacitación y la retención del personal sanitario en los países en desarrollo, especialmente en los países menos adelantados y los pequeños estados insulares en desarrollo; reforzar la capacidad de todos los países, en particular los países en desarrollo, en materia de alerta temprana, reducción de riesgos y gestión de los riesgos para la salud nacional y mundial ${ }^{3}$.

Si se revisa cada uno de los 16 objetivos, descritos más arriba, estos tienen que ver directa o indirectamente con el tercero que está referido a la salud y el bienestar de las personas, siendo la persona el fin supremo. En ese sentido, la salud y el bienestar dependen de esos dieciséis determinantes, que si se desarrollan armónicamente como es el propósito los ODS, se logrará realmente una buena salud y el bienestar.

Por otro lado, los cambios en la población mundial como el incremento de la expectativa de vida, así como la transición epidemiológica con el incremento de las enfermedades crónico degenerativas y la emergencia y reemergencia de enfermedades infecciosas propiciadas por las desastres naturales y el cambio climático, deben hacernos pensar en la necesidad de contar con estrategias de prevención y control de estas enfermedades, tanto trasmisibles como no transmisibles ${ }^{4}$ para abordar el reto del objetivo 3 de los ODS.

De manera muy simple podríamos decir que cuando se habla de salud pública, debe considerarse la salud individual o recuperativa -cuando la persona enferma y debe recuperar su salud-, y la salud colectiva que tiene que ver más con las medidas de prevención y promoción para que las poblaciones no se vean masivamente afectadas y que los servicios puedan responder adecuadamente y oportunamente a la demanda que sea inevitable enfrentar, pese a las medidas de prevención y control que se haya adoptado o no. En ese contexto, debemos mencionar la pertinencia de mantener ese enfoque de salud individual y salud colectiva, de modo que las estrategias de prevención y control se incluyan en la organización del sistema de salud, de los servicios, así como en su dinámica cotidiana, contando con los recursos humanos, los recursos financieros y materiales, en los diferentes ámbitos territoriales y niveles de organización de los servicios, buscando una real integración en los ámbitos nacional, regional y local.

Dado que ahora afrontamos tanto enfermedades transmisibles como no transmisibles, al enfrentar estos problemas sanitarios -ahora y como antes- es necesario discutir lo relacionado a los programas de control tanto de enfermedades transmisibles y no transmisibles que incluyen acciones de prevención; así, a través del tiempo se han implementado programas verticales, luego horizontales, con diferentes resultados. Estos programas de control pueden ser muy efectivos en controlar un daño específico, pero pueden tener efectos nocivos al sistema de salud si son excesivamente verticales y no integrados a los servicios de salud. Pueden generar inequidades y brechas en la calidad de atención si se disponen de más o menos recursos, o el tiempo que se dedique a la labor preventiva o prestacional el personal que muchas veces es insuficiente, sobre todo en el primer nivel de atención. En el otro extremo, si hay una integración completa de los programas en los servicios de salud, es un riesgo cuando los sistemas de salud son débiles en su organización y en su capacidad de gestión. Ambos enfoques se necesitan mutuamente pues los programas de control contribuyen al objetivo general del sistema de salud que es reducir la incidencia y mortalidad por enfermedades, pero no son suficientes para alcanzar un estado de salud integral en las personas. Por eso es importante discutir la forma de integración de los programas de control y de los servicios de salud en un contexto específico, de manera que no se debiliten las acciones de uno o de otro y se alcance un mejor estado de salud de las personas. Esto pasa desde el diseño de la organización, su funcionamiento y la asignación de recursos.

Buscando la complementaridad entre las entidades formadoras (universidades) y las entidades que demandan de recursos humanos para acciones sanitarias, consideramos que un aspecto importante y a la vez un reto es la formación de especialistas que dirijan las estrategias para el control de enfermedades, así como el de la formación de recursos humanos técnicos y operativos para implementar las medidas adoptadas, como la iniciativa para la formación en salud pública a nivel técnico operativo en miembros del servicio militar voluntario ${ }^{5}$. Hace falta sistematizar las medidas de control según el perfil epidemiológico, que se puede conseguir a través de una formación téorica y de campo que muy bien puede ser una acción sinérgica entre las universidades y el estado.

Si concebimos que las medidas de prevención y control en poblaciones es tan importante como la recuperación de la salud perdida, debe haber un equilibrio en la dotación de los recursos para ambas acciones sanitarias que hagan viable una atención oportuna y de calidad, que conjuntamente con una armónica acción intersectorial de los otros ODS, podremos avanzar en ese ansiado tercer objetivo de garantizar una vida sana y promover el bienestar para todos en todas las edades.

\section{REFERENCIAS BIBLIOGRÁFICAS}

1. Naciones Unidas [Internet]. Objetivos de Desarrollo del Milenio, Informe de 2015. Naciones Unidas; 2018 [Fecha de acceso 27 de junio de 2018]. Disponible en: http://www.un.org/es/millenniumgoals/ pdf/2015/mdg-report-2015_spanish.pdf

2. Naciones Unidas [Internet]. Transforming our world: the 2030 Agenda for Sustainable Development. Naciones Unidas; 2018 [Fecha de acceso 27 de junio de 2018]. Disponible en: https:// sustainabledevelopment.un.org/post2015/transformingourworld

3. Organización Mundial de la Salud OMS [Internet] Objetivos de Desarrollo Sostenible: Metas. Ginebra: OMS; 2018 [Fecha de acceso 27 de junio de 2018]. Disponible en: http://www.who.int/topics/ sustainable-development-goals/targets/es/

4. ClubEnsayos. El perfil Epidemiológico y la transición epidemiológica en México y sus determinantes sociales. ClubEnsayos; 2018 [Fecha de acceso 27 de junio de 2018]. Disponible en: https://www.clubensayos.com/AcontecimientosSociales/El-perfil-Epidemiológico-y-la-transiciónepidemiológica/4108259.html

5. Romaní-Romaní F, Curisinche Rojas M, Rojas Arteaga N, Riega Lopez P, Cabezas C. Experiencia intersectorial para reducir la brecha del recurso humano en salud pública a nivel técnico operativo en miembros del servicio militar voluntario del Perú. Rev Peru Med Exp Salud Publica. 2016;33(4):8118. DOI:10.17843/rpmesp.2016.334.2569 